\title{
Global Stability of COVID-19 Model: A Case Study of Nepal
}

\author{
Gauri Bhuju ${ }^{1}$, Ganga Ram Phaijoo ${ }^{2}$, Dil Bahadur Gurung ${ }^{2}$ \\ ${ }^{1}$ Department of Mathematics, Bhaktapur Multiple Campus, \\ Bhaktapur, Nepal \\ ${ }^{1,2}$ Department of Mathematics, School of Science \\ Kathmandu University, Dhulikhel, Nepal \\ Correspondence to: Gauri Bhuju, Email: gauri.bhuju@student.ku.edu.np
}

\begin{abstract}
COVID-19 (Corona Virus Disease) is continuously spreading all over the world from January 2020. It has been the major public health concern worldwide. In Nepal, the confirmed cases of the disease are increasing day by day. Mathematical modeling is one of the best tool to study the transmission dynamics of COVID - 19. In the present work, the transmission dynamics of COVID - 19 in Nepal with isolation is studied by using epidemic compartmental model. The global stability of the equilibrium points of the model are discussed with Lyapunov function. The stability of the disease is dependent on both transmission rate of the disease and the progression rate of the infectious state to isolated or hospitalized state. Simulations are made to observe situation of the disease in Nepal using the mathematical results graphically.
\end{abstract}

Keywords: COVID-19, Compartmental model, Basic reproduction number, Global stability, Lyapunov function

DOI: https://doi.org/10.3126/jnms.v3i2.33954

\section{Introduction}

The first outbreak of COVID-19 occurred in Wuhan City of China in December 2019. After few months it spread worldwide rapidly. On 11 March 2020, World Health Organization (WHO) declared COVID-19 as a pandemic [24]. More than 8 million active positive cases and more than 39 million total positive cases are reported in the world on October 18, 2020. More than 29 million people have recovered and more than 1 million deaths are reported [25]. Due date, more than 39 thousand active positive cases and more than 132 thousand total positive cases of COVID-19 in Nepal [11.

On 23 January 2020, the first case of COVID-19 was reported in Nepal [1, 11. The Government of Nepal announced first lockdown on 24 march 2020 to control the disease transmission and ended on 21 July 2020 and from $3^{\text {rd }}$ June, partial lock down was continued. But transmission of the disease of COVID19 was continued in Nepal because of the lack of awareness about the disease. The local transmission started from 4 April 2020 and more than 100 people were infected each day from 25 April 2020 [11. The transmission continued to increase and on 18 August 2020, more than 1000 people were infected. To control the transmission of the disease, government of Nepal again implemented the lockdown from 19 August to 9 September 2020, only in the most infected cities. However, lockdown is not the permanent solution of this outbreak and it cannot be implemented for long period of time. It has negative impact on the economic condition of Nepal. Also, it will initiate the huge economic problem for daily wage earners, large migrated population and others. Except lockdown, every individual has to take his/her own responsibility, they have to use face mask on gathering, maintain social distance, wash hand regularly, keep themselves away from social gathering and unnecessary movement. We should continue these works in the future until vaccine of the disease is discovered.

Corona viruses are transmitted from person to person through respiratory droplets of an infected person coughs, sneezes, exhales, and close contact with one another [23. The best way to reduce the spread of the disease is use of face mask, regular hand wash, social distancing, mass testing, isolation for confirmed cases, lockdown and quarantine of suspected cases by contact-tracing. Since the researchers have not identified the exact behavior of this new virus, it is one of the best way to study the disease transmission mathematically. Consequently, it is needful to study the transmission dynamics of the disease with different mathematical 
models and to simulate using the available data. All over the world, researchers have used the several mathematical models to study the COVID-19 dynamics [5, 15, 16, 17, 18, 21].

Mathematical models are useful to study the transmission dynamics of infectious disease and to conduct control strategies against the disease 3 , 4, 8, 9, 12, 13, 14. J. Singh et al. used the successive approximation method for short term forecast of infected population in India [18. Y. Li et al. established the time series models based on different mathematical formulas according to the variation law of the original data of Wuhan city [9]. Y. Souleiman et al. studied the local and global stability and bifurcation analysis of equilibrium to examine its epidemiological relevance [19]. Some researchers have studied transmission dynamics of COVID-19 in different cities of the world mathematically and statistically.

One of the potential cause of outbreak of COVID-19 is human to human contact, and isolation of infected person is a best way to reduce the risk of COVID-19 spread. So, in the present work, we have used a mathematical epidemiological model SIHR (Susceptible-Infected-Hospitalized-Recovered) of COVID-19 including isolation to investigate the dynamics of the disease. We have compared this model with the current situation of COVID-19 cases of Nepal. We have discussed local and global stability of the equilibrium points and validated the model by using the COVID-19 cases of Nepal.

\section{Model Formulation and Description}

In the model, total population $N(t)$ is divided into four classes: Susceptible: $C_{S}(t)$, Infected (Infectious): $C_{I}(t)$, Hospitalized: $C_{H}(t)$ and Recovered: $C_{R}(t)$. So, $N(t)=C_{S}(t)+C_{I}(t)+C_{H}(t)+C_{R}(t)$. We have not considered human mobility and it is assumed that the recovery from the disease gives total immunity. Also, for all compartments per day infection rate, recovery time are fixed, and natural death rate are considered same for all the compartments. All the hospitalized individuals are isolated and do not come in contact with susceptible individual. We do not consider the quarantine and the whole population of Nepal is considered to be susceptible.

The mathematical model of the transmission of COVID-19 is described as follows:

$$
\begin{aligned}
\frac{d C_{S}}{d t} & =\pi-\frac{\beta}{N} C_{S} C_{I}-\mu C_{S} \\
\frac{d C_{I}}{d t} & =\frac{\beta}{N} C_{S} C_{I}-(k+\eta+\mu) C_{I} \\
\frac{d C_{H}}{d t} & =k C_{I}-(\delta+\gamma+\mu) C_{H} \\
\frac{d C_{R}}{d t} & =\eta C_{I}+\gamma C_{H}-\mu C_{R} \\
N=C_{S}+C_{I}+C_{H}+C_{R} \quad \text { and } \quad \frac{d N}{d t} & =\pi-\delta C_{H}-\mu N
\end{aligned}
$$


State variables and model parameters are described below.

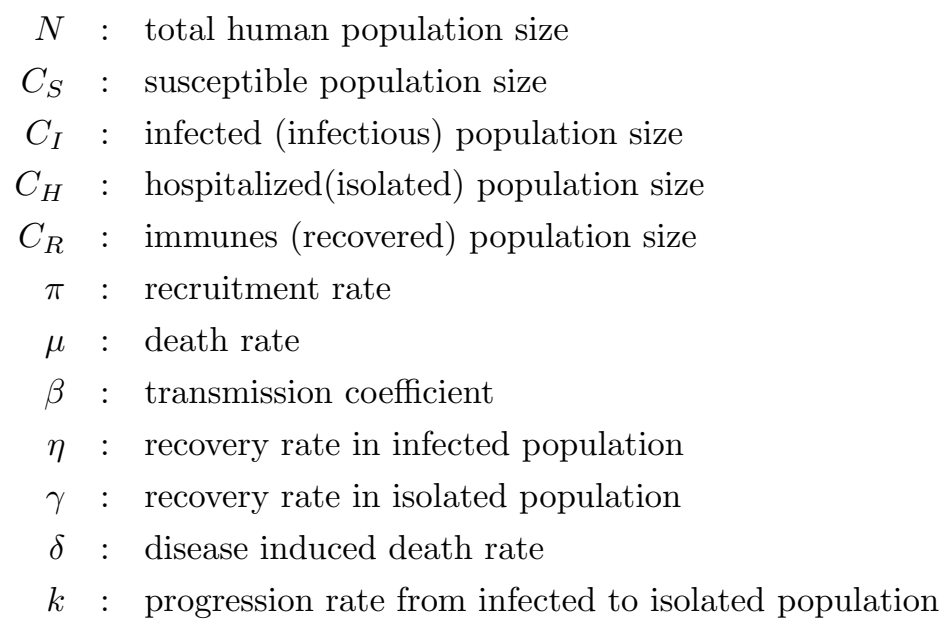

\subsection{Non-negativity and boundedness}

Suppose, $M=\left\{\left(C_{S}, C_{I}, C_{H}, C_{R}\right) \in \mathbb{R}^{4}: 0 \leq C_{S}, C_{I}, C_{H}, C_{R}\right\}$. Then, $M$ should be positively invariant. The solution of the system; the state variables $C_{S}, C_{I}, C_{H}, C_{R}$ cannot exit $M$ by crossing the boundaries $C_{S}=0, C_{I}=0, C_{H}=0, C_{R}=0$.

From the system (1), we have,

$$
N=C_{S}+C_{I}+C_{H}+C_{R} .
$$

Thus,

$$
\begin{aligned}
\frac{d N}{d t} & =\pi-\mu N-\delta C_{H} \leq \pi-\mu N \\
N & \leq \frac{\pi}{\mu}\left(1-e^{-\mu t}\right)+N(0) e^{-\mu t}
\end{aligned}
$$

Hence,

$$
\limsup _{t \rightarrow \infty} N \leq \frac{\pi}{\mu}
$$

Therefore, $C_{S}(t), C_{I}(t), C_{H}(t)$ and $C_{R}(t)$ are bounded above by $\frac{\pi}{\mu}$ on $[0, d)$ for some $d>0$. Hence, for some $d>0$ the solution of the system (1) are bounded on $[0, d)[20$.

\subsection{Existence and uniqueness}

Assume that, the initial conditions of the system are as follows

$$
C_{S}(0)>0, C_{I}(0)>0, C_{H}(0) \geq 0, C_{R}(0) \geq 0
$$

Let $x(t)=\left(C_{S}(t), C_{I}(t), C_{H}(t), C_{R}(t)\right) \in \mathbb{R}^{4}$. The system (1) is written in the form $x^{\prime}=w(x)$ with $w_{i}$, $i=1,2,3,4$ are the components of the vector field $w$, which consists of the algebraic polynomials of state variables. Thus $w_{i}$ are continuous autonomous functions on $\mathbb{R}^{4}$ and partial derivatives $\frac{\partial w_{i}}{\partial C_{S}}, \frac{\partial w_{i}}{\partial C_{I}}, \frac{\partial w_{i}}{\partial C_{H}}$, $\frac{\partial w_{i}}{\partial C_{R}}$ exist and are continuous. Hence by Existence and Uniqueness Theorem, for any initial condition $x(0) \in \mathbb{R}^{4}$ a unique solution of the system $x^{\prime}=w(x)$ exists $[22$. 


\section{Equilibrium Points and Basic Reproduction Number}

There are two equilibrium points in the system of equations (1); the disease free equilibrium point $E_{0}=$ $\left(\frac{\pi}{\mu}, 0,0,0\right)$ and endemic equilibrium point $E_{1}=\left(C_{S}^{*}, C_{I}^{*}, C_{H}^{*}, C_{R}^{*}\right)$.

Moreover, basic reproduction number $R_{0}$ is defined as the average number of secondary infections caused by single infectious individual during his/her entire infectious life time [6, 7]. $F$ and $V$ respectively denote the matrix of transmission terms and the matrix of transition terms of the system (1) at $E_{0}$. $R_{0}$ is defined as the spectral radius of the matrix $F V^{-1}$ i.e., $\rho\left(F V^{-1}\right)$ and is obtained by using the Next Generation Matrix Method [6, 7, 13. Thus, the basic reproduction number at $E_{0}$ is

$$
R_{0}=\frac{\pi \beta}{N \mu(k+\eta+\mu)}
$$

The disease free equilibrium point always exists when $R_{0}<1$ and endemic equilibrium point always exists when $R_{0}>1$.

The endemic equilibrium point $E_{1}=\left(C_{S}^{*}, C_{I}^{*}, C_{H}^{*}, C_{R}^{*}\right)$, where

$$
\begin{aligned}
C_{S}^{*} & =\frac{N(k+\eta+\mu)}{\beta}, & C_{I}^{*}=\frac{N \mu}{\beta}\left(R_{0}-1\right) \\
C_{H}^{*} & =\frac{N \mu k\left(R_{0}-1\right)}{\beta(\delta+\gamma+\mu)}, & C_{R}^{*}=\frac{\gamma N\left(R_{0}-1\right)\left(\pi \beta+\mu^{2} N R_{0}\right)}{\beta(\delta+\gamma+\mu)}
\end{aligned}
$$

\section{Local stability}

Theorem 4.1. (Local Stability of DFE) Disease free equilibrium point of the system of equations (1) is locally asymptotically stable if $R_{0}<1$ and unstable if $R_{0}>1$.

Proof. About $E_{0}$, the Jacobian matrix for the system of equations (1) is in the block matrix,

$$
M=\left[\begin{array}{cc}
U_{1} & U_{2} \\
0 & F-V
\end{array}\right]
$$

If all the eigenvalues of $M$ have negative real parts, then $E_{0}$ is asymptotically stable 7 . Since $M$ is upper triangular matrix, eigenvalues of $M$ are those of $U_{1}$ and $F-V$. Two eigenvalues of matrix $U_{1}$ are $-\mu,-\mu<0$. Now, stability of the disease free equilibrium depends on the eigenvalues of $F-V$ where $F$ is non-negative and $V$ is non-singular $M$-matrix [2].

The eigenvalues of $F-V$ are $-(k+\eta+\mu)<0$ and $(k+\eta+\mu)\left(R_{0}-1\right)$. Thus, the eigenvalues of $F-V$ are negative if $R_{0}<1$ and positive if $R_{0}>1$ [2]. It shows that, $E_{0}$ is locally asymptotically stable if $R_{0}<1$ and unstable if $R_{0}>1$

Theorem 4.2. The endemic equilibrium point $E_{1}=\left(C_{S}^{*}, C_{I}^{*}, C_{H}^{*}, C_{R}^{*}\right)$ is stable if $R_{0}>1$.

Proof. Two eigenvalues of the Jacobian matrix $J$ for the system of equations (1) about $E_{1}$ are $\lambda_{1}=-\mu<0$ and $\lambda_{2}=-q<0$. Other two eigenvalues are roots of the quadratic equation

$$
\lambda^{2}-c \lambda+d=0
$$

where, $c=-\frac{\pi \beta}{p}<0$ and $d=\mu p\left(R_{0}-1\right)$.

Here, $c<0$; and $d>0$ if $R_{0}>1$. Thus, two roots of the equation have negative real parts if $R_{0}>1$. Therefore, all the eigenvalues of $J$ have negative real parts if $R_{0}>1$. Hence, the equilibrium point $E_{1}$ is stable if $R_{0}>1$. 
Journal of Nepal Mathematical Society(JNMS), Vol. 3, Issue 2(2020); G.Bhuju, G.R.Phaijoo, D.B.Gurung

\section{Global stability}

Let $x^{\prime}=w(x)$ where $w: \mathbb{R}^{n} \rightarrow \mathbb{R}$, be an autonomous system of ordinary differential equation with an equilibrium $x^{*}$.

Definition $5.1([10])$. . Let $V: \mathbb{R}^{n} \rightarrow \mathbb{R}$,

1. A scalar function $V(x)$ is called radially unbounded if $V(x) \rightarrow \infty$ and $\|x\| \rightarrow \infty$.

2. Let $V$ be a continuous scalar function. It is called positive definite on the entire space if

(a) $V\left(x^{*}\right)=0$

(b) $V(x)>0$ for $x \neq x^{*}$

Theorem 5.1 (10]). (Lyapunov's Stability Theorem) If a function $V(x)$ is globally positively definite and radially unbounded and its time derivative is globally negative,

$$
V^{\prime}(x)<0 \quad \forall x \neq x^{*}
$$

Then the equilibrium $x^{*}$ is globally stable.

Definition $5.2([10])$. . If a function $V(x)$ exists that satisfies the condition of Lyapunov's stability theorem then the function is called a Lyapunov function.

Theorem $5.2([10])$. (Krasovkil- LaSalle Theorem (Extension of Lyapunov's Theorem )) Consider the autonomous system $x^{\prime}=w(x)$, where $x^{*}$ is an equilibrium, that is $w\left(x^{*}\right)=0$. Suppose, there exists a continuously differentiable function $V: \mathbb{R}^{n} \rightarrow \mathbb{R}$ and that this function is positive definite on the entire space and radially unbounded and that is satisfies

$$
V^{\prime}(x) \leq o \quad \forall t \quad \text { and } \quad \forall x \in \mathbb{R}^{n}
$$

Define the invariant set $P=\left\{x \in \mathbb{R}^{n} \mid V^{\prime}(x)=0\right\}$. If $P$ contains only the equilibrium $x^{*}$ is globally stable.

Theorem 5.3. (Global Stability of DFE) Assume $R_{0}<1$. Then the disease free equilibrium is globally asymptotically stable.

Proof. We have $C_{R}=N-C_{S}-C_{I}-C_{H}$, the system (1) as follows;

$$
\begin{aligned}
\frac{d C_{S}}{d t} & =\pi-\frac{\beta}{N} C_{S} C_{I}-\mu C_{S} \\
\frac{d C_{I}}{d t} & =\frac{\beta}{N} C_{S} C_{I}-p C_{I} \\
\frac{d C_{H}}{d t} & =k C_{I}-q C_{H}
\end{aligned}
$$

where $p=k+\mu+\eta$ and $q=\delta+\gamma+\mu$.

We know that if disease free equilibrium $E_{0}^{*}=\left(\frac{\pi}{\mu}, 0,0\right)$ is globally stable, then $R(t) \rightarrow 0$ and $E_{0}$ for the system (1) is globally stable.

Consider a Lyapunov function 10 .

$$
V=\alpha\left(C_{S}-C_{S}^{*}-C_{S}^{*} \ln \frac{C_{S}}{C_{S}^{*}}\right)+\frac{1}{p} C_{I}+\frac{1}{k} C_{H}
$$

where $\alpha>0$ to be determined and, $C_{S}^{*}=\frac{\pi}{\mu}$.

At $E_{0}^{*}, V=0$. 
At first we have to show that $V>0$ for all $\left(C_{S}, C_{I}, C_{H}\right) \neq\left(\frac{\pi}{\mu}, 0,0\right)$.

Since $\frac{1}{p} C_{I}>0$ and $\frac{1}{k} C_{H}>0$, it is sufficient to show that

$$
\alpha C_{S}^{*}\left(\frac{C_{S}}{C_{S}^{*}}-1-\ln \frac{C_{S}}{C_{S}^{*}}\right)>0 .
$$

We have, the function $h(x)=x-1-\ln x$ has global minimum at $x=1$ and $h(1)=0$.

Hence, $h(x)>0, \quad \forall x>0$ and $x \neq 1$.

So, the first term of $V$ is positive. Thus, $V$ is positively definite at $E_{0}^{*}$. Again, since as $t \rightarrow \infty, V \rightarrow \infty, V$ is radially unbounded.

Now differentiating $V$ with respect to $t$ and using (3), we get

$$
\begin{aligned}
\frac{d V}{d t} & =\alpha\left(1-\frac{C_{S}^{*}}{C_{S}}\right) \frac{d C_{S}}{d t}+\frac{1}{p} \frac{d C_{I}}{d t}+\frac{1}{k} \frac{d C_{H}}{d t} \\
& =\alpha\left(1-\frac{C_{S}^{*}}{C_{S}}\right)\left(\pi-\frac{\beta}{N} C_{S} C_{I}-\mu C_{S}\right)+\frac{1}{p}\left(\frac{\beta}{N} C_{S} C_{I}-p C_{I}\right)+\frac{1}{k}\left(k C_{I}-q C_{H}\right) \\
& =2 \alpha \pi-\frac{\beta}{N} C_{S} \alpha C_{I}-\alpha \mu C_{S}-\frac{\alpha \pi^{2}}{\mu C_{S}}+\frac{\alpha \pi \beta}{\mu N} C_{I}+\frac{\beta}{N p} C_{S} C_{I}-\frac{q}{k} C_{H}
\end{aligned}
$$

If we choose $\alpha=\frac{1}{p}$, then

$$
\frac{d V}{d t}=-\alpha \pi\left(\frac{\mu C_{S}}{\pi}+\frac{\pi}{\mu C_{S}}-2\right)+\frac{\beta \pi}{\mu N p} C_{I}-\frac{q}{k} C_{H}
$$

Since from equilibrium equation, we have

$$
k C_{I}=q C_{H}
$$

Therefore

$$
\frac{d V}{d t}=-\alpha \pi\left(\frac{\mu C_{S}}{\pi}+\frac{\pi}{\mu C_{S}}-2\right)+\left(R_{0}-1\right) C_{I}
$$

The last term is negative, since $R_{0}<1$. Let $\frac{\pi}{\mu C_{S}}=a$, then we have the first term is

$$
a+\frac{1}{a}-2=\frac{(a-1)^{2}}{a}>0 \quad \text { if } \quad a \neq 1
$$

So, $a+\frac{1}{a}-2>0$ for all $a>0$ and $a \neq 1$.

Hence, we have

$$
V^{\prime}<0 \quad\left(C_{S}, C_{I}, C_{H}\right) \neq E_{0}^{*}
$$

Therefore, by Lyapunov's theorem, the disease free equilibrium point $E_{0}^{*}$ is globally asymptotically stable.

Theorem 5.4. Assume $R_{0}>1$. Then, the endemic equilibrium is globally asymptotically stable.

Proof. Consider the system of equation (3). Consider a Lyapunov function [10]

$$
V=\left(C_{S}-C_{S}^{*}-C_{S}^{*} \ln \frac{C_{S}}{C_{S}^{*}}\right)+\left(C_{I}-C_{I}^{*}-C_{I}^{*} \ln \frac{C_{I}}{C_{I}^{*}}\right)+\frac{k+\eta+\mu}{k}\left(C_{H}-C_{H}^{*}-C_{H}^{*} \ln \frac{C_{H}}{C_{H}^{*}}\right)
$$

Here, $V=0$ when $\left(C_{S}, C_{I}, C_{H}\right)=\left(C_{S}^{*}, C_{I}^{*}, C_{H}^{*}\right)$, otherwise $V>0 . V$ is also radially unbounded. Now it is remained to show that $\frac{d V}{d t}<0$. 
$\underline{\text { Journal of Nepal Mathematical Society(JNMS), Vol. 3, Issue 2(2020); G.Bhuju, G.R.Phaijoo, D.B.Gurung }}$

Differentiating $V$ with respect to $t$ and using (1), we get

$$
\begin{aligned}
\frac{d V}{d t} & =\left(1-\frac{C_{S}^{*}}{C_{S}}\right) \frac{d C_{S}}{d t}+\left(1-\frac{C_{I}^{*}}{C_{I}}\right) \frac{d C_{I}}{d t}+\frac{p}{k}\left(1-\frac{C_{H}^{*}}{C_{H}}\right) \frac{d C_{H}}{d t} \\
& =\left(1-\frac{C_{S}^{*}}{C_{S}}\right)\left(\pi-\frac{\beta}{N} C_{S} C_{I}-\mu C_{S}\right)+\left(1-\frac{C_{I}^{*}}{C_{I}}\right)\left(\frac{\beta}{N} C_{S} C_{I}-p C_{I}\right)+\frac{p}{k}\left(1-\frac{C_{H}^{*}}{C_{H}}\right)\left(k C_{I}-q C_{H}\right)
\end{aligned}
$$

From equilibrium equation we have

$$
\pi=\frac{\beta}{N} C_{S}^{*} C_{I}^{*}+\mu C_{S}^{*}
$$

We get,

$$
\begin{aligned}
\frac{d V}{d t}= & -\frac{\mu}{N}\left(C_{S}^{*}-C_{S}\right)^{2}+\frac{\beta}{N} C_{S}^{*} C_{I}^{*}-\frac{\beta}{N} \frac{C_{S}^{*^{2}}}{C_{S}} C_{I}^{*}+\frac{\beta}{N} C_{S}^{*} C_{I}-\frac{\beta}{N} C_{S} C_{I}^{*}+p C_{I}^{*}-\frac{1}{k} p q C_{H} \\
& -p C_{I}^{*}\left(\frac{C_{I} C_{H}^{*}}{C_{I}^{*} C_{H}}\right)+\frac{1}{k} p q C_{H}^{*} .
\end{aligned}
$$

Again, we have from equilibrium equation,

$$
\begin{aligned}
k C_{I}^{*} & =q C_{H}^{*} \text { and } \frac{\beta}{N} C_{S}^{*} C_{I}^{*}=p C_{I}^{*} \\
\Longrightarrow \frac{1}{k} p q C_{H}^{*} & =p C_{I}^{*}
\end{aligned}
$$

We get,

$$
\frac{d V}{d t}=-\frac{\mu}{N}\left(C_{S}^{*}-C_{S}\right)^{2}+\frac{\beta}{N} C_{S}^{*} C_{I}^{*}\left(3-\frac{C_{S}^{*}}{C_{S}}-\frac{C_{S}}{C_{S}^{*}}-\frac{C_{I} C_{H}^{*}}{C_{I}^{*} C_{H}}\right)
$$

Clearly, the first term of right hand side of (4) is negative unless $C_{S}=C_{S}^{*}$. In the second term, we have to show that $\left(3-\frac{C_{S}^{*}}{C_{S}}-\frac{C_{S}}{C_{S}^{*}}-\frac{C_{I} C_{H}^{*}}{C_{I}^{*} C_{H}}\right)$ is non-positive. For this, suppose

$$
a_{1}=\frac{C_{S}^{*}}{C_{S}}, \quad a_{2}=\frac{C_{S}}{C_{S}^{*}}, \quad a_{3}=\frac{C_{I} C_{H}^{*}}{C_{I}^{*} C_{H}}
$$

The Geometric Mean of the sequence is

$$
\sqrt[3]{a_{1} a_{2} a_{3}}=\sqrt[3]{\left(\frac{C_{I} C_{H}^{*}}{C_{I}^{*} C_{H}}\right)}>0 \quad \text { if } \quad R_{0}>1
$$

The Arithmetic Mean of the sequence is

$$
\frac{a_{1}+a_{2}+a_{3}}{3} .
$$

Since,

$$
\begin{aligned}
\text { A.M. } & \geq \text { G.M. } \\
\Longrightarrow a_{1}+a_{2}+a_{3} & \geq 3 \sqrt[3]{\left(\frac{C_{I} C_{H}^{*}}{C_{I}^{*} C_{H}}\right)}>3 \quad \text { whenever, } \sqrt[3]{\left(\frac{C_{I} C_{H}^{*}}{C_{I}^{*} C_{H}}\right)}>1
\end{aligned}
$$

Thus, the second term is also non-positive, whenever $\sqrt[3]{\left(\frac{C_{I} C_{H}^{*}}{C_{I}^{*} C_{H}}\right)}>1$. Hence,

$$
\frac{d V}{d t} \leq 0
$$


Now, we apply the Krasovkii-LaSalle Theorem. Consider a set

$$
U=\left\{x \in \mathbb{R}^{n} \mid V^{\prime}(x)=0\right\}
$$

$\frac{d V}{d t}=0$ if and only if $C_{S}=C_{S}^{*}$ and

$$
\left(\frac{C_{S}^{*}}{C_{S}}+\frac{C_{S}}{C_{S}^{*}}+\frac{C_{I} C_{H}^{*}}{C_{I}^{*} C_{H}}\right)=3
$$

Since $C_{S}=C_{S}^{*}$, then $\frac{d C_{S}}{d t}=0$. From the system (1) we get $C_{I}=C_{I}^{*}$.

Finally, we get from (5), $C_{H}^{*}=C_{H}$. Therefore, the set $U$ consists only one element $\left(C_{S}^{*}, C_{I}^{*}, C_{H}^{*}\right)$. Hence, the theorem is proved.

\section{Model Validation and Discussion}

Figure 1 illustrates the daily reported data of COVID-19 cases of Nepal [11. It contains total confirmed cases, active infectious cases, recovered cases and death cases due to COVID-19 infection from March 23 to October 16, 2020. With some rational assumptions and parameter estimation, we have fitted the reported data of active infectious cases and recovered cases from August 1 to October 16, 2020 in Nepal to our model in figures 2 and 3

Figure 4 shows that active infectious population of Nepal are increased with increasing values of $\beta$. So, the basic reproduction number is increasing with increasing the transmission rate of the disease (figure 5 ). But it is decreasing with the increase in the progression rate of number of population from infectious to isolation or hospitalization and recovery rate of disease (figures 6 and 7). Thus, for the global stability of the disease free equilibrium, we need to reduce the transmission rate of the disease. Together with this, we need to progress the recovery rate and increase the isolation for the infected.

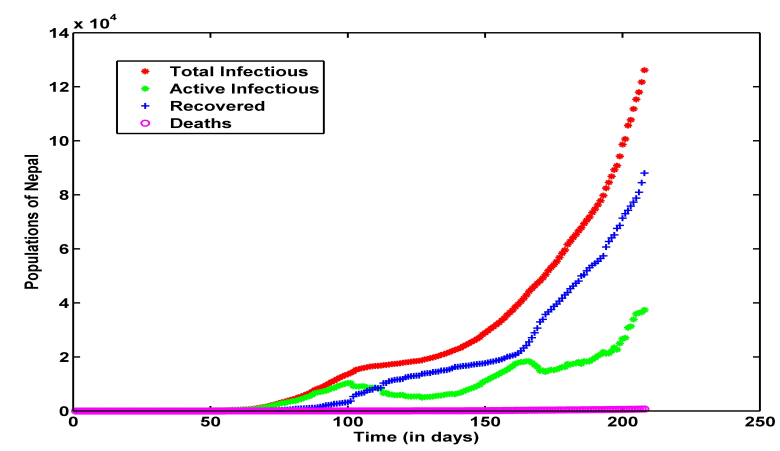

Figure 1: Reported cumulated data of COVID-19 in Nepal from March 23 to October 16, 2020.

\section{Conclusions}

Based on the transmission dynamics of COVID-19 in Nepal, we constructed a time dependent simple SIHR mathematical model. We computed the disease free equilibrium point, endemic equilibrium point and basic reproduction number of the model. Also, we discussed about the local and global stability of the disease at the equilibrium points. The disease free equilibrium point is locally and globally stable when $R_{0}<1$ and unstable when $R_{0}>1$. Further, the endemic equilibrium point is locally and globally stable when 


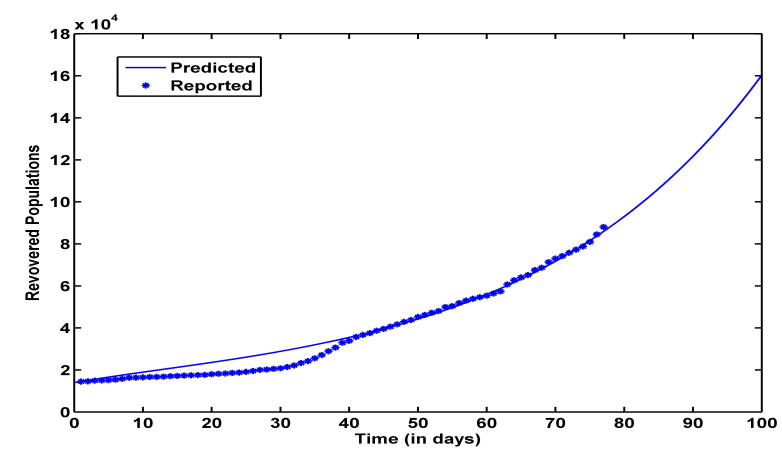

Figure 2: Reported and Predicted Recovered Cases of COVID-19 in Nepal from August 1 to October 16 , 2020 .

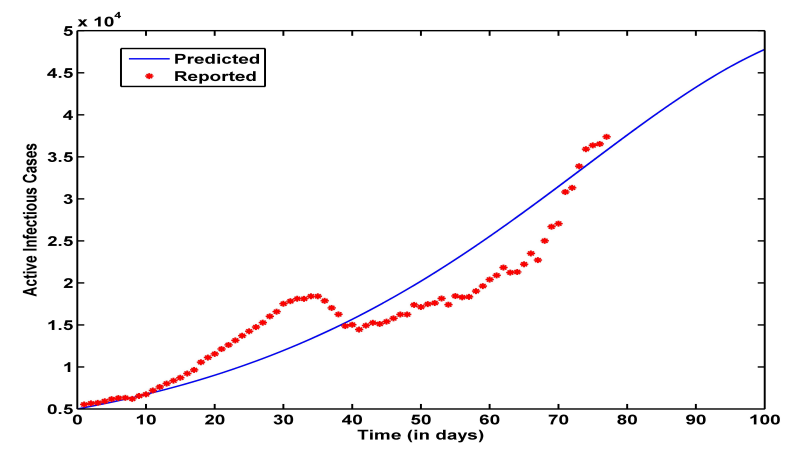

Figure 3: Reported and Predicted Recovered Cases of COVID-19 in Nepal from August 1 to October 16 , 2020.

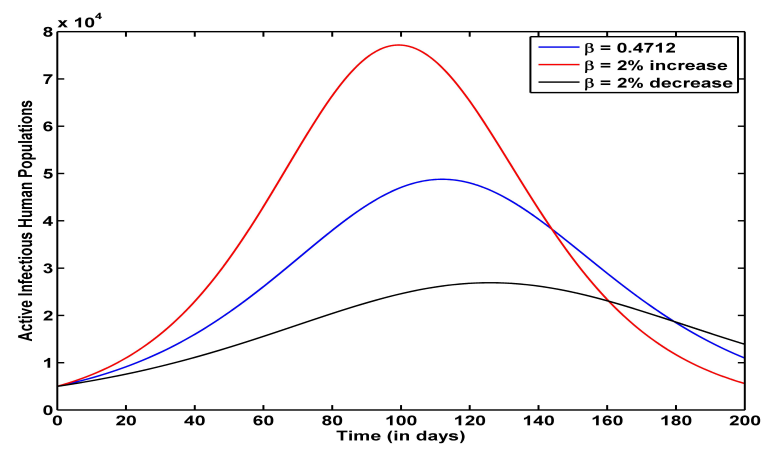

Figure 4: Active infectious population with different values of $\beta$.

$R_{0}>1$. Thus, for the stability of disease COVID-19, we need to get $R_{0}<1$ using different types of control measures such as social distancing, self-isolation, testing facilities, face mask wearing etc.

We used the model to fit the active infectious cases and recovered cases of COVID-19 in Nepal from August to October 2020. We estimated and predicted the values of the parameters of the model and to get reasonable match between the reported cases and predicted cases of infected and recovered population of COVID-19 from August 1 to October 16, 2020 in Nepal. Also, we compared the basic reproduction number $R_{0}$ with the transmission rate $\beta$, progression rate from infected to isolation $k$ and recovery rate $\eta$ 


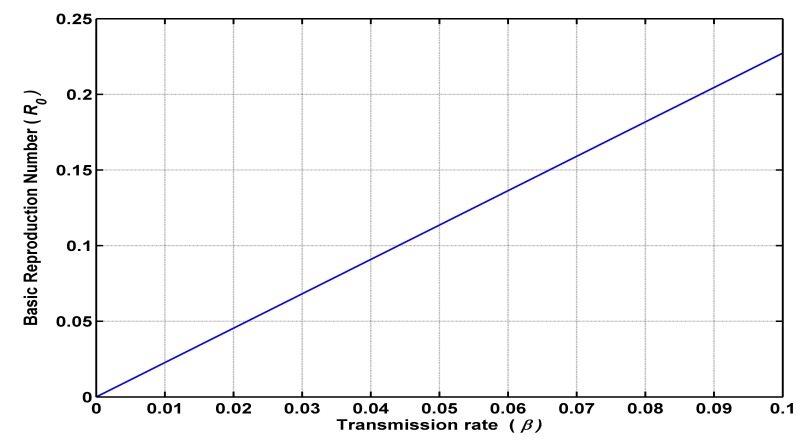

Figure 5: Basic reproduction number with transmission rate of disease.

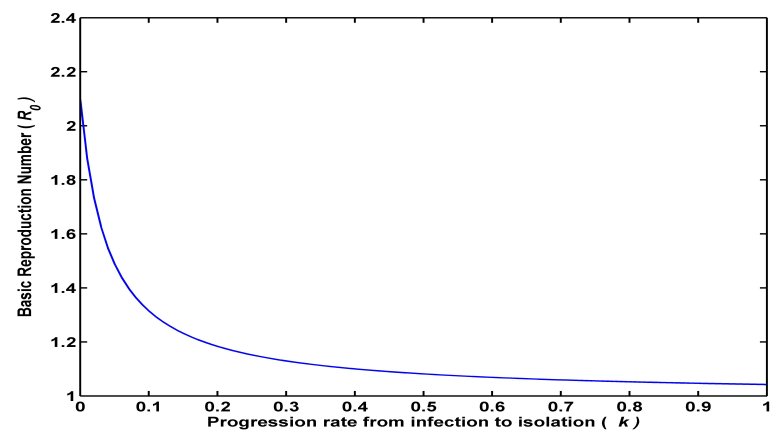

Figure 6: Basic reproduction number with progression rate of disease from infection to isolation.

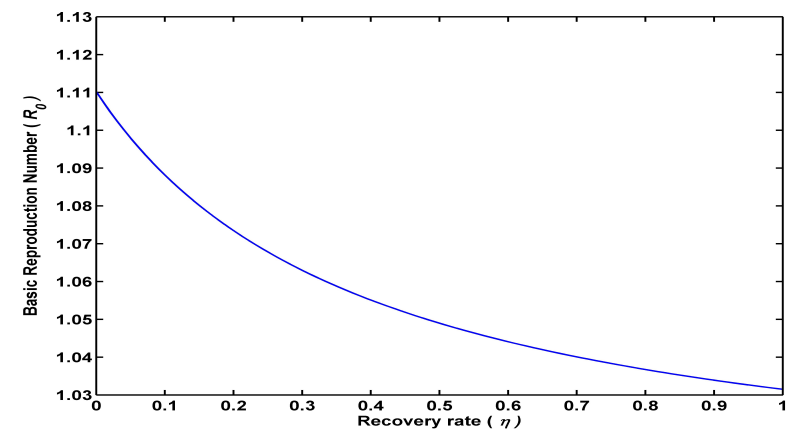

Figure 7: Basic reproduction number with recovery rate of the disease.

in the cases of Nepal. $R_{0}$ increases with $\beta$ and decreases with increase the values of $k$ and $\eta$. Thus, for the global stability of the COVID-19 in Nepal, we need to decrease the transmission rate using the different control measures. Also, we have to improve the recovery rate, and progression rate of infected to isolation or hospital. Thus, to manage the pandemic of COVID-19 the government of Nepal need to promote the public health measures with improving the isolation wards together with using different control measures for preventing the transmission of the disease. 
Journal of Nepal Mathematical Society(JNMS), Vol. 3, Issue 2(2020); G.Bhuju, G.R.Phaijoo, D.B.Gurung

\section{References}

[1] Bastola, A., Sah, R., Morales, A. J. R. and Chu, D., 2020, March, The first 2019 novel coronavirus in Nepal, National Library of medicine, 20(3), 279 - 280.

[2] Berman, A. and Plemmons, R. J., 1979, Nonnegative matrices in mathematical sciences, Computer Science and Applied Mathematics, Academic press, New York.

[3] Bhuju, G., Phaijoo, G. R. and Gurung, D. B., 2018, Mathematical study on impact of temperature in malaria disease transmission dynamics, Advances in Computer Sciences 1(2), 1 - 8.

[4] Bhuju, G., Phaijoo, G. R. and Gurung, D. B., 2020, Fuzzy approach analyzing SEIR-SEI dengue dynamics, BioMed Research International 2020, 1 - 11.

[5] Bhuju, G., Phaijoo, G. R. and Gurung, D. B., 2020, Sensitivity analysis of COVID-19 transmission dynamics, International Journal of Advanced Engineering Research and Application (IJA-ERA) 6(4), $72-82$.

[6] Diekmann, O., Heesterbeek, J. A. P. and Metz, J. A. J., 1990, On the definition and computation of basic reproduction ratio $R_{0}$ in models for infectious diseases in heterogeneous populations, Journal of Mathematical Biology 28(4), 365 - 382.

[7] Driessche, P. and Watmough, J., 2002, Reproduction number and sub-threshold endemic equilibria for compartmental models for disease transmission, Mathematical Biosciences 180, 29 - 48.

[8] Kermack, W. O. and McKendrick, A. G., 1991, Contribution to the mathematical theory of epidemic, Bulletin of Mathematical Biology 53, 33 - 55.

[9] Li, Y., Wang, B., Peng, R., Zhan, C., Zhan, Y., Liu, Z., Jiang, X. and Zhao, B., 2020, Mathematical modeling and epidemic prediction of COVID-19 and its significance to epidemic prevention and control measures, Annals of Infectious Disease and Epidemiology 5(1).

[10] Martcheva, M., 2010, An introduction of mathematical epidemiology, Texts in Applied Mathematics, Springer, 61 .

[11] MoHP, 2020, Nepal's latest update on COVID-19, Ministry of Health And Population, Kathmandu, Nepal.

[12] Phaijoo, G. R. and Gurung, D. B., 2017, Mathematical model of dengue disease transmission dynamics with control measures, Journal of Advances in Mathematics and Computer Science 23, 1 - 12.

[13] Phaijoo, G. R. and Gurung, D. B., 2016, Mathematical study of dengue disease transmission in multipatch environment, Applied Mathematics and Mathematical Bioscience 7, 1521 - 1533.

[14] Phaijoo, G. R. and Gurung, D. B., 2018, Sensitivity analysis of SEIR-SEI model of dengue disease, GAMS Journal of Mathematics and Mathematical Bioscience 6(a), 41 - 50.

[15] Polonsky, J. A. et al., 2019, Outbreak analytics: a developing data science for informing the response to emerging pathogens, Philosophical Transactions of the Royal Society B.

[16] Quasim, M., 2020, Analysis of the worldwide corona virus (COVID-19) pandemic trend; a modelling study to predict its spread, Cold Spring Harbor Laboratory, BMJ Yale, doi: https://doi.org/10.1101/2020.03.30.20048215.

[17] Quasim, M., Ahmad, W., Zhang, S., Yasir, M., and Azhar, M., 2020, Data model to predict prevalence of COVID-19 in Pakistan,Cold Spring Harbor Laboratory, BMJ Yale, doi: https://doi.org/10.1101/2020.04.06.20055244.

[18] Singh, J., Ahluwalia, P. K. and Kumar, A., 2020, Mathematical model based COVID-19 prediction in India and its different states, Cold Spring Harbor Laboratory, BMJ Yale, doi: https://doi.org/10.1101/2020.05.16.20104232. 
[19] Souleiman, Y., A. Mohamed A. and Ismail, L., June 17, 2020, Analysis the dynamics of SIHR model: COVID-19 case in Djibouti, Mathematics and Computer Science, Applied mathematicsl, 1.

[20] Srivastav A. K. and Ghosh, S. M., 2019, Assessing the impact of treatment on the dynamics of dengue fever: A case study of india, Applied Mathematics and Computation 362, 124533.

[21] Tang, Y. and Wang, S., 2020, Mathematical modeling of COVID-19 in the United States, Emerging Microbes and Infections.

[22] Wiggins, S., 2003., Introduction to applied nonlinear dynamical systems and chaos, Texts in Applied Mathematics, Springer - Verlag New York.

[23] World Health Organization, 29 March 2020, Modes of transmission of virus causing COVID19: implications for IPC precaution recommendations, https://www.who.int/newsroom/commentaries/detail/modes-of-transmission-ofvirus-causing-covid19-implications-foripc-precaution-recommendations.

[24] World Health Organization, 2020, Coronavirus disease (COVID-19) outbreak, https://www.euro.who.int/en/health-topics/health-emergencies/coronaviruscovid19.

[25] World Health Organization, 2020, Situation update, view coronavirus disease 2019 (COVID-19), https://www.worldometers.info/coronavirus. 